\title{
Tribbles homolog 3-mediated targeting the AKT/mTOR axis in mice with retinal degeneration
}

\author{
Irina V. Saltykova ${ }^{1}$, Asif Elahi ${ }^{1}$, Priyam M. Pitale ${ }^{1}{ }^{1}$, Oleg S. Gorbatyuk ${ }^{1}$, Mohammad Athar ${ }^{2}$ and Marina S. Gorbatyuk ${ }^{1}{ }^{凶}$
}

(c) The Author(s) 2021

Various retinal degenerative disorders manifest in alterations of the AKT/mTOR axis. Despite this, consensus on the therapeutic targeting of mTOR in degenerating retinas has not yet been achieved. Therefore, we investigated the role of AKT/mTOR signaling in rd16 retinas, in which we restored the AKT/mTOR axis by genetic ablation of pseudokinase TRB3, known to inhibit phosphorylation of AKT and mTOR. First, we found that TRB3 ablation resulted in preservation of photoreceptor function in degenerating retinas. Then, we learned that the mTOR downstream cellular pathways involved in the homeostasis of photoreceptors were also reprogrammed in $r d 16 \mathrm{TRB}^{-/-}$retinas. Thus, the level of inactivated translational repressor $\mathrm{p}-4 \mathrm{E}-\mathrm{BP} 1$ was significantly increased in these mice along with the restoration of translational rate. Moreover, in $r d 16$ mice manifesting decline in p-mTOR at P15, we found elevated expression of Beclin-1 and ATG5 autophagy genes. Thus, these mice showed impaired autophagy flux measured as an increase in LC3 conversion and p62 accumulation. In addition, the RFP-EGFP-LC3 transgene expression in rd16 retinas resulted in statistically fewer numbers of red puncta in photoreceptors, suggesting impaired late autophagic vacuoles. In contrast, TRIB3 ablation in these mice resulted in improved autophagy flux. The restoration of translation rate and the boost in autophagosome formation occurred concomitantly with an increase in total Ub and rhodopsin protein levels and the elevation of E3 ligase Parkin1. We propose that TRB3 may retard retinal degeneration and be a promising therapeutic target to treat various retinal degenerative disorders.

Cell Death and Disease (2021)12:664; https://doi.org/10.1038/s41419-021-03944-w

\section{INTRODUCTION}

Inherited retinal degeneration (IRD) comprises a heterogeneous group of ocular diseases caused by mutations in more than 260 genes identified to date. The disease manifests as degeneration of rods (e.g., retinitis pigmentosa) and cones (cone-rod dystrophy), primarily due to mutations in rod- or cone-specific genes. This occurs in addition to mutations in genes expressed in retinal pigment epithelial (RPE) cells, which lead to photoreceptor degeneration (e.g., Leber's congenital amaurosis [LCA]) [1].

Previous work identified common signaling pathways in retinal degeneration in various animal models regardless of the affected genes or mutations. The study also indicated that reprogramming a metabolism towards anabolic processes for biosynthesis may be a novel therapeutic strategy for photoreceptor neuroprotection during acute stress [2]. Therefore, strategies that boost the anabolic rate and meet photoreceptor demands during retinal degeneration could generate a therapeutic platform independent of affected genes and mutations.

One common pathway affected in degenerated retinas is the altered protein kinase $B(A K T) /$ mammalian target of the rapamycin (mTOR) axis. A serine/threonine protein kinase mTOR exists in distinct mTORC1 and mTORC2 complexes. Together with AKT, mTOR plays a critical role in a wide spectrum of cellular signaling, including regulation of autophagy, lysosome biogenesis, energy metabolism, protein and lipid synthesis, cytoskeleton organization, and cell survival. Debates continue on the therapeutic targeting of the AKT/ mTOR axis under conditions associated with aging, neurodegenerative disorders, cancer, and diabetes, and whether the activation or inhibition of mTOR should be taken as a therapeutic approach is currently under investigation. While researchers are still searching for an answer to this question, they agree that the severity, the stage of the disease, and the variety of affected tissues and cells should be taken into consideration in solving this puzzle. Thus, recent work has reviewed the mTOR signaling pathway, altering it under different neurodegenerative conditions and emphasizing the complexity of mTOR targeting [3]. For example, in Alzheimer's disease, mTOR inhibition is beneficial for degenerating neuronal cells because this approach leads to stimulation of autophagy necessary for aberrant protein degradation. In contrast, another study on the therapeutic potential of L-DOPA treatment for patients with Parkinson's disease found that mTOR hyperactivation brings benefits to neuronal cell survival $[4,5]$.

Another example is a study on Huntington disease (HD), in which a decrease in mTOR activation associated with sequestrated Huntingtin protein $(\mathrm{Htt})$ aggregates in postmortem HD brains was found $[6,7]$. Lee and colleagues provided evidence that introducing the constitutively active form of the MTORC1 activator Ras homolog enriched in brain (RHEB) into HD mouse brains alleviates

\footnotetext{
${ }^{1}$ Department of Optometry and Vision Science, School of Optometry, University of Alabama at Birmingham, Birmingham, AL, USA. ${ }^{2}$ Department of Dermatology, School of Medicine, University of Alabama at Birmingham, Birmingham, AL, USA. ${ }^{凶}$ email: mgortk@uab.edu Edited by N. Bazan.
} 
mitochondrial dysfunction, aberrant cholesterol homeostasis, striatal atrophy, and impaired dopamine signaling and increases autophagy.

In the vision science field, mTOR signaling has also generated interest as a therapeutic target. However, similar to the central nervous system neurodegeneration research field, there is a lack of consensus on the therapeutic targeting of mTOR for different retinal degenerative disorders. Moreover, it has been demonstrated that depending on the insult, the same retinal cell type could respond to the same treatment in a different way. For example, the death of retinal ganglion cells (RGC) known to occur through apoptosis during the course of glaucoma and diabetic retinopathy is differently regulated by mTOR-mediated autophagy, and the role of autophagy may differ depending on the triggering injury and the relevant cell death pathway [8]. Phosphorylated mTOR ( $p$-mTOR) is significantly downregulated in both diabetic and glaucomatous retinas [8]. However, depending on whether or not p-AMPK is involved in the retinal pathogenesis, the results of mTOR-mediated regulation of autophagy can vary. Chronic intraocular pressure elevation results in autophagic cell death without AMRK involvement, which is not the case for the changes in the diabetic retina [8]. Therefore, in this study, the authors concluded that IOP elevation was associated with autophagy activation, resulting in RGC death, while in the diabetic retina, the AMPK-mediated autophagy induction may serve as a survival attempt of RGCs.

Indeed, multiple studies with animal and cellular models of different retinal degenerative diseases have revealed that the targeting of mTOR does not lead to a straightforward cell fate. An investigation conducted with RPE cells deficient in oxidative phosphorylation, mimicking molecular events occurring in agerelated macular degeneration (AMD), demonstrated that the early RPE changes associated with hypertrophy and dedifferentiation coincide with robust activation of the mTOR pathway [9]. Therefore, to combat AMD, it is necessary to attempt to downregulate mTOR activity. Overall, these results confirmed a previous finding demonstrating that downregulation of mTORC could delay the aging process in the RPE cells [10] and enhance cellular degradation and self-renewal [11]. In mice with oxygeninduced retinopathy, modeling aberrant retinal vascular development, targeting mTOR activity through either rapamycin or VEGFmediated signaling revealed that, in endothelial cells, the mTOR pathway is activated by VEGF. This finding also demonstrated that mTOR contributes to physiologic vascular development and is modulated in a postnatal, age-dependent manner [12].

Despite the convincing data on the therapeutic effect of mTOR downregulation in these studies, another line of research proposes that mTOR activation could be neuroprotective for degenerating retinal cells. The group led by Dr. Rajala demonstrated that a constitutively active version of mTOR mutant promotes cone survival in mice with RP in a nutrition-independent manner [13]. This study confirmed previous findings from Cepko's and Punzo's laboratories indicating that cone cell death in mice with IRD occurs as a result of the downregulation of insulin/mTOR signaling. These laboratories demonstrated that the cellautonomous activation of mTORC1 delays photoreceptor cell death in animal models of retinal degeneration [14-17]. In support of these findings, a recent study conducted with the tuberous sclerosis complex 1 (Tsc1) gene, an mTOR inhibitor, demonstrated that silencing this gene enhanced photoreceptor survival and increased retinal function in PDE6 $b^{\mathrm{H} 620 \mathrm{Q} / \mathrm{H} 620 \mathrm{Q}}$ mice [18].

Despite the significance of the studies conducted with animal models of ocular degenerative diseases, the answer to questions about whether a uniform strategy could be applied in a variety of IRD models remains elusive. Therefore, the disagreement about the role of mTOR in controlling IRD fueled our interest in this molecule in rd16 mice, mimicking LCA in humans.

In our study, the rd16 mice demonstrated severe retinal degeneration accompanied by $\mathrm{p}-\mathrm{AKT} / \mathrm{p}-\mathrm{mTOR}$ downregulation at
P15 [19]. The role of $p-A K T / p-m T O R$ in the degenerating retinas can be studied by modulating its expression, activity, or degradation. Although mTOR inhibitors have been recently identified due to their ability to reduce proliferation in cancers, the development of mTORC1 activators that are beneficial for retinal degeneration [14-17] or other diseases is still a critical need. While many commercially available mTOR inhibitors, including rapamycin, dactolisib, everolimus, etc., are on the market, MHY1485 is the only current potent cell-permeable activator, whose specificity and efficiency in agonizing mTOR activity in vivo still need to be determined. Therefore, we decided to take a genetic approach to reprogram the AKT/mTOR signaling in $r d 16$ mouse photoreceptors by ablating a pseudokinase tribbles homolog 3 (TRB3 or TRIB3), a protein kinase-like scaffold with impaired catalytic activity, known to inhibit phosphorylation of AKT [20] and mTOR [21].

Integrated stress response (ISR) that is persistently activated in rd16 mice [19] has been reported to induce TRB3 overexpression via ATF4 [22]. TRB3 participates in a variety of cellular signaling. Thus, TRB3 has been proposed to regulate cell death, stress responses, inflammation, cell differentiation, and protein degradation [23]. It also functions by controlling autophagy flux and protein degradation [23]. TRB3 acts as a metabolic switcher controlling GLUT1 activity and glucose uptake [24]. Therefore, we decided to investigate the TRB3-mediated control of AKT/mTOR signaling in the retina of mice with IRD. We demonstrated that the restoration of the AKT/mTOR axis slows down IRD progression and delays photoreceptor cell death. To our knowledge, this is the first study that has validated TRB3 as a therapeutic target in animal models of retinal degeneration; this study emphasizes the critical need to generate a TRB3-based cellular therapy to retard inherited retinal dystrophies.

\section{MATERIAL AND METHODS \\ Animals}

BXD24/TyJ-Cep290rd16/J (rd16) and C57BL/6J mice were obtained from Jackson Laboratory (Bar Harbor, ME). TRB3 ${ }^{-1-}$ mice were generated as previously described [25]. All mice used in the study experiments were on C575BL/6 background. The $r d 16$ mice (Jackson Laboratory) were crossed with $\mathrm{TRB}^{-/-}$mice to produce $r d 16 \mathrm{TRB}^{-1-}$ genotype-carrying mice. The mice were housed in the UAB animal core facility with a $12 \mathrm{~h}$ light/dark cycle and free access to a standard diet and water. Sample sizes ranged from three to five. Retinas from control and experimental mice of both sexes were used at postnatal days (P) 15, 18, or 20. Mice were euthanized by $\mathrm{CO}_{2}$ asphyxiation followed by cervical dislocation. All animal experiments followed a protocol (IACUC\#131109793) approved by the University of Alabama at Birmingham Institutional Animal Care and Use Committee (IACUC) and conformed to guidelines from the Association of Research in Vision Science and Ophthalmology.

\section{Electroretinography (ERG)}

ERG was performed using the LKC BIGSHOT ERG instrument. Mice were dark-adapted overnight and were anesthetized with an intra-peritoneal injection of ketamine/xylazine, based on their weight, and placed on a $37^{\circ} \mathrm{C}$ heating pad. After pupil dilation with topical $2.5 \%$ phenylephrine (Paragon BioTeck, Inc., 42702-102-15), a drop of Gonak solution (AKORN, Lake Forest, IL) was applied, and a monopolar contact loop was placed on the surface of the cornea. Needle electrodes were placed under the scalp and in the tail to serve as a reference and as a ground electrode, respectively. The mice were then exposed to five flashes of $25 \mathrm{~cd} \mathrm{~s} / \mathrm{m}^{2}$ in $45 \mathrm{~s}$ intervals, and ERGs were recorded $(n=3-7)$. The ERG waveforms were then analyzed using LKC EM software.

\section{Histology}

Eyes were enucleated at $\mathrm{P} 15,18$, or 20 and placed in $4 \%$ paraformaldehyde (PFA) for $20 \mathrm{~min}$ on ice. After $30 \mathrm{~min}$, a needle (33G) was inserted at the limbus to create a small hole for eye tissue fixation in $4 \%$ paraformaldehyde. After $1 \mathrm{~h}$ of incubation, the eyes were washed with PBS and immersed in $30 \%$ sucrose overnight. The eyes were cryopreserved in an optimal cutting temperature (OCT) compound (VWR: 25608-930) and kept 
at $-80^{\circ} \mathrm{C}$ until sectioning. Cryomolds were equilibrated to the temperature inside $\left(-21^{\circ} \mathrm{C}\right)$ the cryostat sectioning system (Leica CM 1510S; Leica, Buffalo Grove, IL, USA). Twelve micromolar retinal sections were prepared using a cryostat tissue sectioning system. The sections were stained with hematoxylin and eosin (Electron Microscopy Sciences: 26754-1A, 26762$01)$. The nuclei of photoreceptors were then counted by a masked-toresults investigator $(n=4-5)$.

For immunohistochemistry, retinal sections were incubated with primary anti-RHO (1D4) antibody (University of British Columbia) overnight at $4{ }^{\circ} \mathrm{C}$ in a humidity chamber, followed by washing and incubation for $1 \mathrm{~h}$ at room temperature with secondary antibodies. Sections were counterstained with DAPI (Vector Laboratories, H-1200). Images were collected with an Olympus FluoView-1000 confocal microscope.

To count the red puncta cells in the retinas, the C56BL/ $6^{\text {RFP-GFP-LC3 }}$ mice were crossed with $r d 16$ and $r d 16$ TRB $^{-1-}$ to obtain $r d 16^{\text {RFP-GFP-LC3 }}$ and $r d 16$ TRB3 $^{-/-R F P-G F P-L C 3}$ mice. The puncta become GFP-negative/mRFP-positive (red) upon fusion with lysosomes [26]. At P15, these mice were sacrificed and perfused with 4\% PFA, and their eyeballs were nucleated and immersed in $30 \%$ sucrose overnight. The next day, the eyeballs were cryopreserved in OCT medium and sectioned to prepare $20-\mu \mathrm{M}$ retinal sections. Confocal fluorescent microscopy was applied to make the images, focusing on the inner segments (IS) of photoreceptors to count the red puncta. A blind-to-results investigator calculated the red puncta in IS of photoreceptors, evidencing the presence of normally functioning lysosomes with acidic $\mathrm{pH}(n=5-7)$.

\section{Analysis of nascent protein synthesis}

The SUnSET method has been described previously [27]. Briefly, mice were intraperitoneally injected with puromycin (puromycin dihydrochloride: Santa Cruz Biotechnology, CAS 58-58-2) at a dosage of $0.04 \mu \mathrm{mol} / \mathrm{g}$ body mass. We then sacrificed the mice and harvested their retinas to prepare protein extracts with RIPA buffer. Total protein $(40 \mu \mathrm{g}$ per well) was electrophoresed on a 4-12\% sodium dodecyl sulfate (SDS)-polyacrylamide gradient gel (Bio-Rad, Hercules, CA, USA) and blotted onto a PVDF. The membranes were then incubated with an antibody specific to puromycin (MABE343, mouse) and processed with a secondary antibody specific to IgG2a (goat anti-mouse peroxidase affinipure lgG, Fcy Subclass 2a Specific: 115-035-206; Jackson Immuno Research Laboratories Inc., West Grove, PA, USA). After scanning the density of the puromycin-incorporated bands using a LI-COR imager, the membranes were stained with Coomassie Brilliant Blue R-250 staining solution (Bio-Rad, \#1610436) for their normalization. The relative densities of entire lanes were measured using ImageJ software $(n=4-6)$.

\section{Cell culture, treatment, and transfection}

A spontaneously immortalized human Müller cell line (developed by Limb et al. in 2002 [28]) was maintained in DMEM (Hyclone, South Logan, UT), supplemented with $1 \mathrm{mM}$ L-glutamine, FBS (10\%; BioAbChem, Ladson, SC), glucose $(4.5 \mathrm{~g} / \mathrm{L})$, penicillin/streptomycin (100 units $/ \mathrm{mL} ; 0.1 \mathrm{mg} / \mathrm{mL}$ ) in a humidified $5 \% \mathrm{CO}_{2}$ incubator at $37{ }^{\circ} \mathrm{C}$. Following incubation, the cells were detached by digestion with $0.25 \%$ trypsin and continually passaged. Lipofectamine 2000 (Invitrogen, CA, USA) was used for transient transfection according to the manufacturer's protocol. Briefly, MIO-M1 cells in 12-well plates were transfected with $3 \mu \mathrm{g}$ of pAAV-CAG-TRIB3-GFP in opti-MEM (Invitrogen, CA, USA). Five hours later, the medium was replaced with fresh cell culture medium containing FBS (10\%) and an antibiotic mixture. Experiments were quadruplicated and repeated three times.

Antisense oligodeoxynucleotides (ASO) were synthesized by Sigma-Aldrich. The ASO sequence against TRB3 (ASO-TRB3) was 5'-GTCC AGTCATCACACAGGCA-3'. The control ASO has the sequence $5^{\prime}$-CCTTCCC TGAAGGTTCCTCC- $3^{\prime}$ and was introduced into cells as a negative control. The first five bases and last five bases of chimeric ASOs have a $2^{\prime}-0-(2-$ methoxy)-ethyl modification. The ASOs were selected based on the data provided by Weismann et al. [29]. For the ASO assay, $12 \mathrm{~h}$ after the transfection of MIO-M1 cells with TRB3 CDNA, the cells were transfected with $3 \mu \mathrm{g}$ of ASO-TRB3 or ASO-control. The cells were collected $48 \mathrm{~h}$ after ASO transfection.

\section{Retinal explants}

Retinas were isolated from each animal. Individual right mouse retinas were incubated in culture media with $50 \mu \mathrm{M}$ chloroquine, while left retinas were incubated without chloroquine for $24 \mathrm{~h}$ at $37^{\circ} \mathrm{C}$ in a humidified cell culture chamber with $5 \% \mathrm{CO}_{2}$. After the treatment retinas were washed in
PBS, retinal protein extracts were prepared and western blot was conducted. Ratios of LC3II/I and p62 in treated vs. untreated retinas were used to analyze the capacity of the retinas to proceed with LC3 I lipidation $(n=6)$ and accumulate p62 $(n=4-5)$.

\section{Immunoblotting and immunoprecipitation}

The mouse retinas were dissected and lysed with RIPA buffer supplemented with $1 \%$ Halt protease and phosphatase inhibitor cocktail (Thermo Fisher Scientific, Waltham, MA). After homogenization, the retina extracts were rotated for $40 \mathrm{~min}$ at $4{ }^{\circ} \mathrm{C}$ and centrifuged at $14,000 \mathrm{~g}$ for $20 \mathrm{~min}$ at $4{ }^{\circ} \mathrm{C}$. The protein concentration of the lysates was estimated using Bio-Rad protein assay (5000001, Hercules, CA, USA). Sample proteins (40-60 $\mu \mathrm{g}$ ) were separated by SDS-PAGE and electroblotted to a PVDF membrane. The following antibodies were used: rabbit anti-p-mTor (D9C2, 5536), rabbit anti-mTOR $(7 C 16,2983)$, rabbit anti-p-AKT (p-S473, 4060), rabbit anti-AKT (4691), rabbit anti-4E-BP1 (53H11, 9644), rabbit anti-p-4E-BP1 (T37/46, 2855), rabbit anti-Beclin-1 (D40C5, 3495), rabbit anti-LC3A/B (D3U4C, 12741), and rabbit anti-Ubiquitin (3933) from Cell Signaling; rabbit-antiLAMP2 (L0668), mouse anti-Parkin (05-882), rabbit anti-Actin (A2066), and mouse anti- $\beta$-Actin (A2228) from Sigma-Aldrich; rabbit anti-SQSTM1/p62 (ab101266) and rabbit anti-UBR1 (ab42420) from Abcam; and TRIB3 (B-2) sc-390242 and TRB-3 (D-4) sc-365842 from Santa Cruz Biotechnology.

The total extracts from eight retinas were immunoprecipitated with Rho 1D4 monoclonal antibody according to the manufacturer's procedures. Briefly, the extracts were incubated at $4{ }^{\circ} \mathrm{C}$ with $5 \mu \mathrm{g}$ of antibody or an equal amount of normal mouse IgG (Millipore Corp., USA) overnight and subsequently with protein A/G agarose beads (Santa Cruz Biotechnology) for $4 \mathrm{~h}$. The beads were washed four times with $1 \times$ PBS. The immunoprecipitated proteins were eluted for $10 \mathrm{~min}$ at $70^{\circ} \mathrm{C}$ with $2 *$ Lammie buffer and further investigated by immunoblotting.

\section{Statistics}

The Student's t-test was used to compare two groups, and one-way ANOVA was carried out to compare three or more groups. All statistics were calculated using Graphpad Prism 8 software.

\section{RESULTS \\ rd16 $\mathrm{TRB}^{-/-}$mice demonstrated an increase in the AKT $\rightarrow$ mTOR axis activity}

mTOR activity is regulated by activated Rheb, growth factors, and amino acid supplementation or by a decrease in the phosphatase activity of the proposed MTMR3 phosphatase, a member of the myotubularin phosphatidylinositol 3-phosphatase family [30]. We decided to use an alternative approach employing genetic ablation of TRB3 to reprogram the p-AKT and p-mTOR levels; TRB3 has been reported to inhibit the activity of both AKT [20] and mTOR [21].

The $r d 16$ mice demonstrated a significant increase in the TRB3 level accompanied by both p-AKT and p-mTOR downregulation at P15-P20 [19]. Therefore, we generated the $r d 16 \mathrm{TRB3}^{-1-}$ mice to monitor the status of the p-AKT/p-mTOR axis in their retinas. Histological and molecular biological assessments of $r d 16 \mathrm{TRB}^{-1-}$ retinas were conducted from P15 to 18 . We learned that TRB3 ablation in degenerating retinas enhances levels of both p-AKT and $\mathrm{p}-\mathrm{mTOR}$ at P15. Furthermore, while no difference in $\mathrm{p}$-AKT levels was observed between $\mathrm{C} 57 \mathrm{BL} / 6$ and $r d 16 \mathrm{TRB}^{-1-}$ retinas (Fig. 1A, B), the decrease in p-AKT level between $r d 16$ and $r d 16$ $\mathrm{TRB}^{-1-}$ was dramatic $(p<0.05$ for comparison between $r d 16$ and $r d 16 \mathrm{TRB}^{-1-}$ ). Reduction in $\mathrm{p}$-mTOR was also detected between C57BL/6 and $r d 16$ retinas confirming previous results [27]. The difference between $r d 16$ and $r d 16 \mathrm{TRB}^{-/-}$measured by one-wayANOVA was not statistically significant.

To confirm the effect of TRB3 on AKT phosphorylation, we next performed an in vitro study with MIO-M1 Müller glial cells. In these cells, we overexpressed mouse TRB3 cDNA and observed a significant $50 \%$ reduction in the $\mathrm{p}-\mathrm{AKT}^{\mathrm{S473}}$ level (Fig. 2A, B). Moreover, when we treated the MIO-M1 cells overexpressing TRB3 with ASO-TRB3, a dramatic reduction in TRB3 level accompanied by the restoration of $p-A K T^{S 473}$ was detected (Fig. 2C, D). These 
A

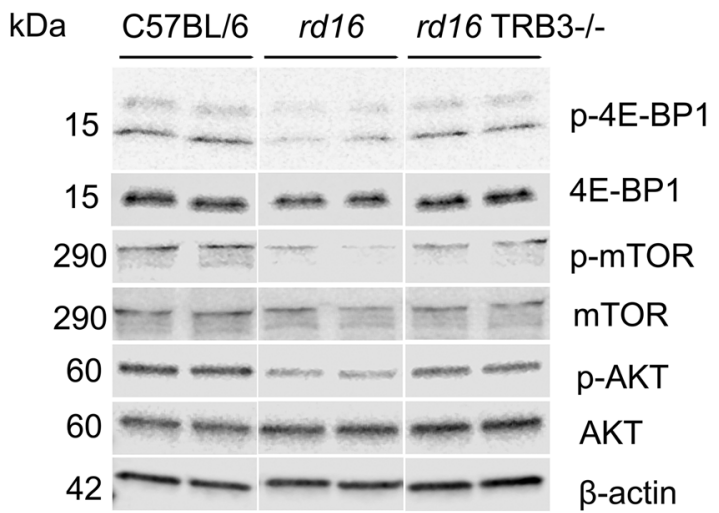

C

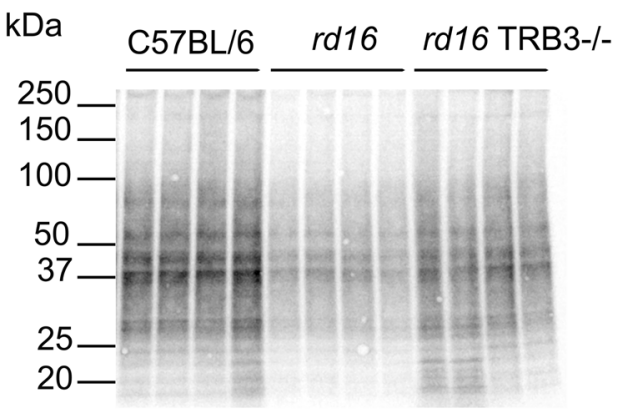

E

Puromycin

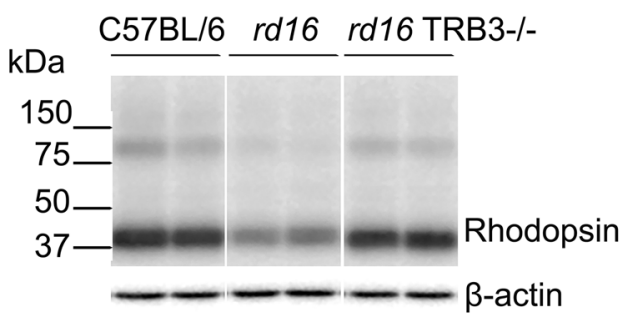

F

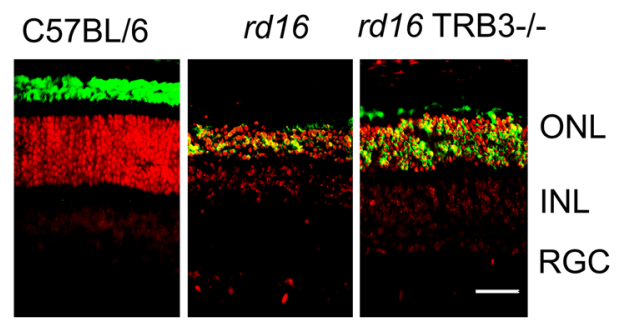

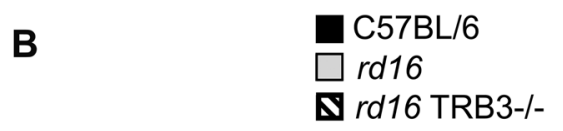

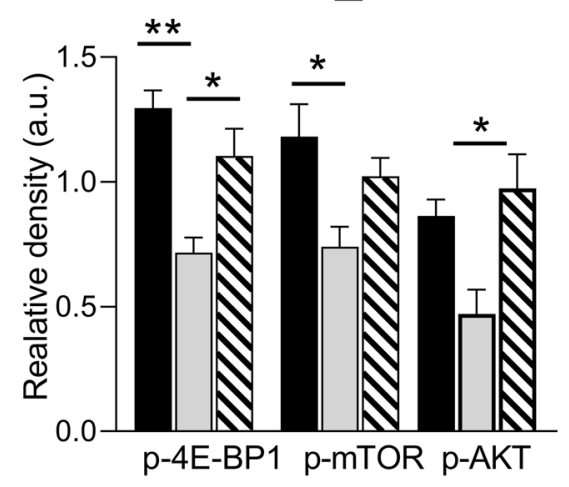

D

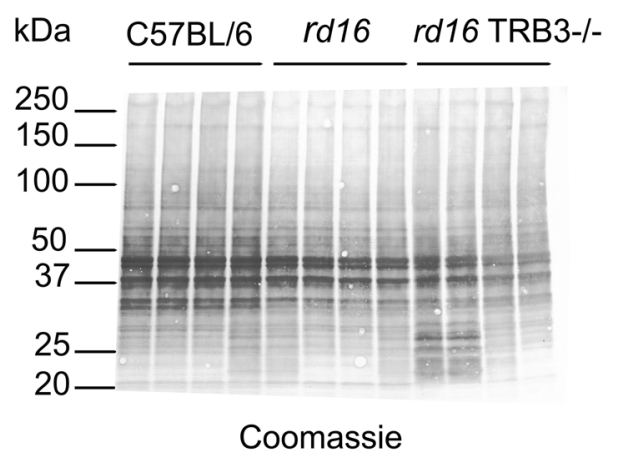

G

C57BL/6

rd16

$\mathbf{\nabla}$ rd16 TRB3-/-

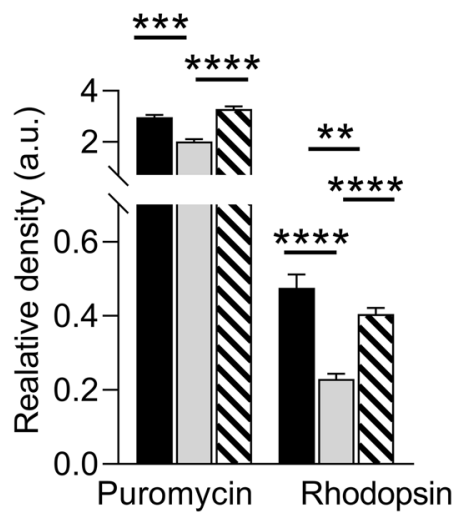

Fig. 1 TRB3 ablation restores the p-AKT/p-mTOR axis in rd16 retinas. A Images of western blot membranes treated with anti-p-4E-BP1, $-4 \mathrm{E}-$ BP1, -p-mTOR, -mTOR, $\mathrm{p}-\mathrm{AKT} \mathrm{T}^{\text {Ser473, }}$-AKT, and -actin primary antibodies and secondary antibodies scanned with LI-COR imager in three groups of mice. B Quantitation of band density of western blot images demonstrates a statistically significant increase in p-4E-BP1 and p-AKT in $r d 16$ $\mathrm{TRB}^{-1-}$ retinas. Increase in p-4E-BP1 was also accompanied by an enhancement of mTOR activity $(n=4)$. C Images of a western blot membrane treated with anti-puromycin antibody to detect the translational level in retinas $(n=4)$. Retinal protein extracts containing incorporated puromycin as the result of intraperitoneal injection in mice were run to detect the density of incorporated puromycin. The density was normalized through protein loading detected by staining with coomassie blue (D). E Retinal protein extracts from P15 C57BL/6, rd16, rd16 TRB3 $^{-1-}$ mice were run on polyacrylamide gel. Western blot images of membranes immunoblotted with anti-Rhodopsin antibody are shown $(n=4)$. F Retinal cryosections of P20 C57BL/6, rd16, and $r d 16 \mathrm{TRB}^{-1-}$ mice were used to perform the IHC with antibody against rhodopsin (in green). Propidium iodide labels the nuclei of the outer nuclear (ONL) and the inner nuclear (INL) layer, and the retinal ganglion cells (RGC). Accumulation of rhodopsin in the ONL was observed in both $r d 16$ and $r d 16$ TRB3 $^{-1-}$ retinas. G Quantitation of the incorporated puromycin in animal retinas to detect translational level (C, D) and rhodopsin protein for western immunoblotting. $\mathbf{E}$ The restoration of the translational rate and an increase in rhodopsin level were observed in $r d 16 \mathrm{TRB}^{-1-}$ retinas $(n=4)$. Data are shown as mean \pm SEM; a.u. $=$ arbitrary units; the scale is $50 \mu \mathrm{m}$. ${ }^{*} p<0.05,{ }^{* *} p<0.01,{ }^{* * *} p<0.001$, and ${ }^{* * *} p<0.0001$. 
A

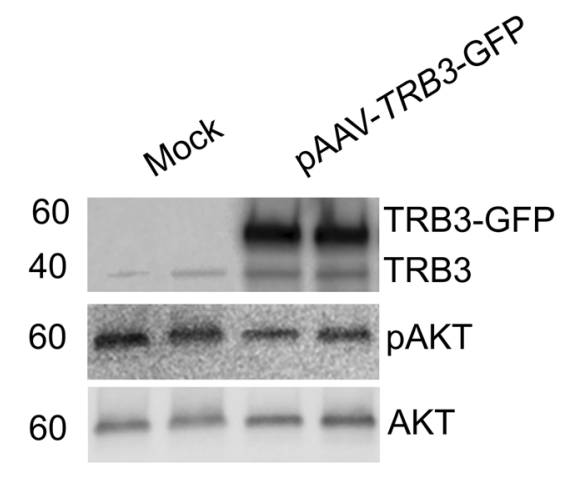

C

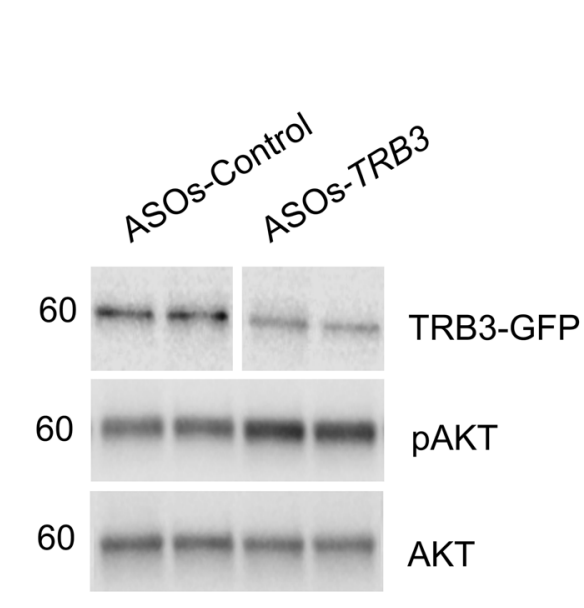

B

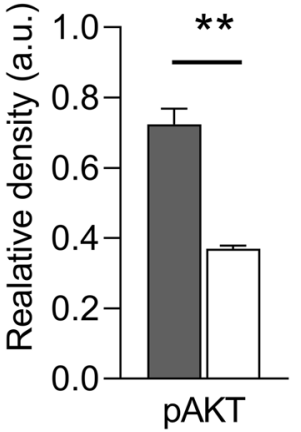

D

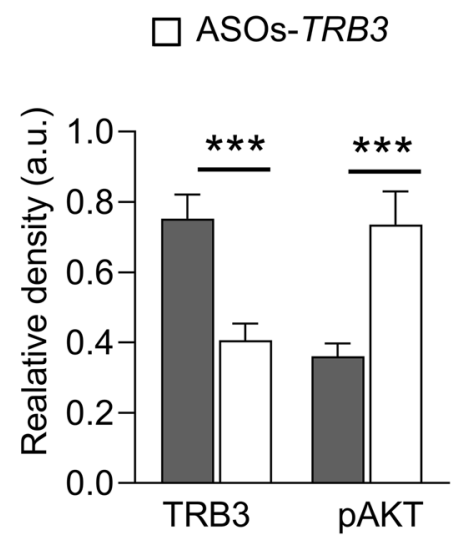

Fig. 2 The impact of TRB3 on the phosphorylation of AKT registered in MIO-M1 cells overexpressing TRB3. A MIO-M1 cells were transfected with AAV-CAG-TRB3-GFP plasmid. Seventy-two hours post-transfection, the cells were harvested, and protein extracts were separated running polyacrylamide gel. Images of western blots stained with anti-TRB3 and anti-pAKT ${ }^{\text {Ser473 }}$ antibodies are shown. B Quantitation of p-AKT normalized to AKT is shown. About a $50 \%$ reduction in p-AKT level was observed $(n=4)$. C MIO-M1 cells were transfected with AAV-CAG-TRB3-GFP plasmid in addition to control and anti-TRB3 ASOs for $48 \mathrm{~h}$. TRB3, p-AKT, and AKT were detected in the protein extracts. D An analysis of densitometry of TRB3 and pAKT ${ }^{\text {Ser473 }}$ bands normalized to the bands for $\beta$-actin and AKT, respectively, is shown $(n=4)$. Data are shown as mean \pm SEM; a.u. = arbitrary units; ${ }^{* *} p<0.01,{ }^{* * *} p<0.001$.

results suggest that in retinal tissues, TRB3 can regulate AKT activity.

\section{TRB3 ablation in $r d 16$ mouse retinas reduced photoreceptor cell death and delayed retinal degeneration}

Histological analyses of $r d 16 \mathrm{TRB}^{-1-}$ retinas demonstrated that TRB3 ablation delayed the death of photoreceptor cells; four more rows of photoreceptor cell nuclei were detected across the retina on average $(p<0.0001)$ at P18 (Fig. 3A, B). The retardation of the death of photoreceptor cells in $r d 16 \mathrm{TRB}^{-1-}$ retinas was also accompanied by increases in the scotopic a- and b-wave ERG amplitudes $(p<$ 0.0001 ). We detected a twofold increase in the a-wave and a fourfold increase in the b-wave amplitudes at P18 (Fig. 3B-D). Altogether, these data suggest that TRB3 ablation delays retinal degeneration in rd16 mice. Therefore, we next decided to analyze whether the recovery of the AKT/mTOR pathway affects downstream signaling in degenerating photoreceptors.

rd16 TRB3 $^{-I-}$ mice manifested a restoration of translational rate and an increase in rhodopsin level

AKT/mTOR signaling is known to participate in a variety of cellular responses, including metabolism and autophagy. Therefore, we next assessed the translation rate known to be attenuated in P15 rd16 retinas [19]. Because 4E-BP1 is known to be phosphorylated by mTORC1 [31], we then decided to check whether the levels of $\mathrm{p}-4 \mathrm{E}-\mathrm{BP} 1$ is altered in $r d 16 \mathrm{TRB}^{-/-}$. We found that the $r d 16 \mathrm{TRB}^{-1-}$ mouse retinas manifested an increase in the p-4E-BP1 level (Fig. 1A, B). This increase implies that the $r d 16 \mathrm{TRB}^{-1-}$ degenerating retinas could have a diminished level of active translational repressor, which interfered with the assembly of the 5-cap mRNA recognition complex. Therefore, using the puromycin-based SUnSET method previously validated in the retina [19], we further learned that the rate of protein synthesis was restored in $r d 16 \mathrm{TRB}^{-1-}$ retinas compared to $r d 16$ and $\mathrm{C} 57 \mathrm{BL} / 6$ retinas (Fig. $1 \mathrm{C}, \mathrm{D}, \mathrm{G}, p<$ $0.001)$. Because of translational restoration and the fact that rhodopsin is the most abundant retinal protein, we next assessed the rhodopsin level in degenerating retinas. We found that a dramatic increase in rhodopsin protein occurred along with the increase in the general translational rate in $r d 16 \mathrm{TRB}^{-/-}$ retinas (Fig. 1E-G, $p<0.001$ ).

rd16 TRB3 - I- retinas manifested restoration of autophagyassociated protein expression and improved autophagy flux Autophagy regulated by mTOR is a major digestion process that removes damaged macromolecules and organelles in cells [32]. To 
A

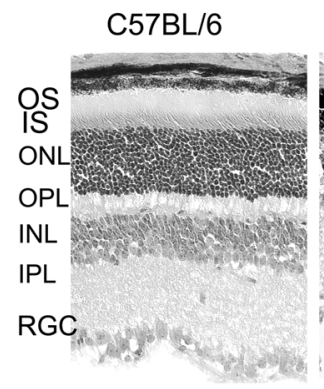

$r d 16$

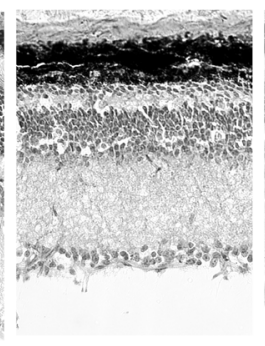

rd16 TRB3-/-

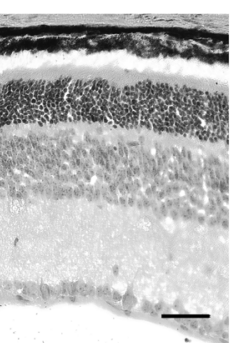

B

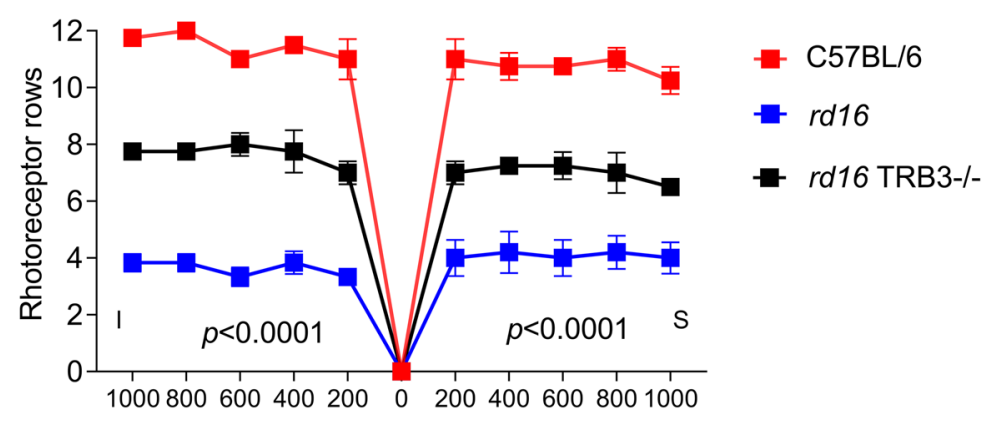

C

D

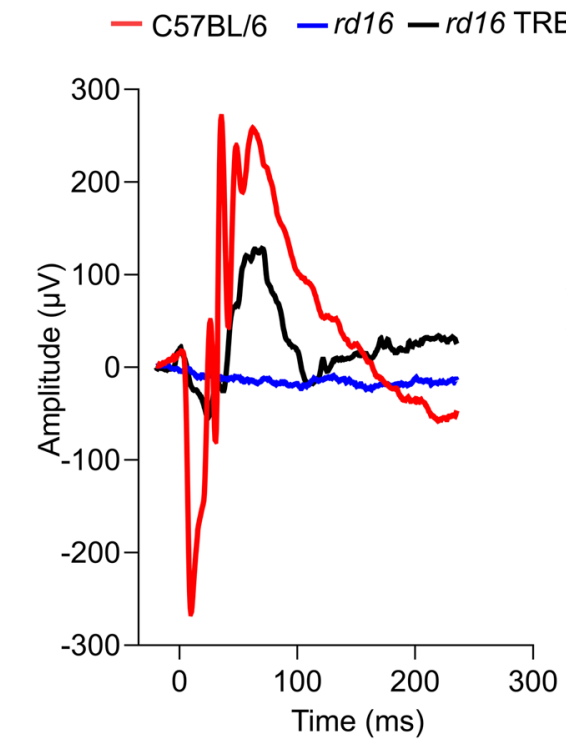

\author{
(1)
}

C57BL/6

$r d 16 \mathbf{N}$ rd16 TRB3-/-

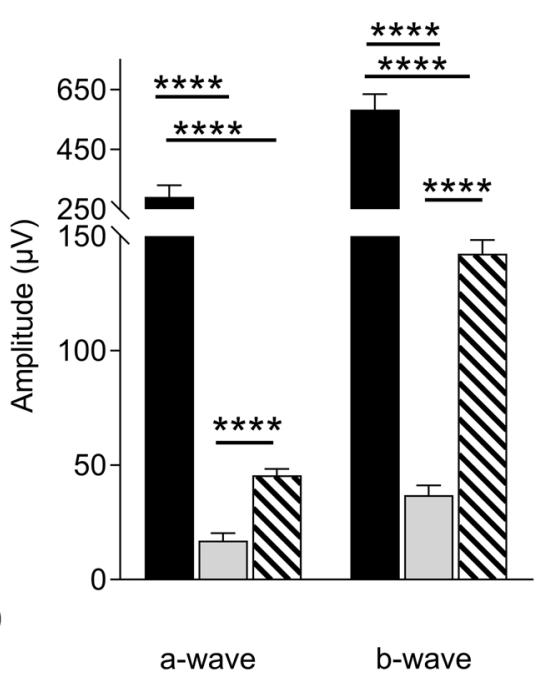

Fig. 3 TRB3 knockout decreases the photoreceptor cell loss detected in P18 $r$ 116 retinas. A Images of C57BL/6, $r d 16$, and $r d 16$ TRB3 ${ }^{-1-}$ retinas stained with eosin and hematoxylin are shown. The scale is $50 \mu \mathrm{m}$. B The retinal images were used to calculate the photoreceptor nuclei across the retinas. Preservation of photoreceptor cells was detected in $r d 16$ TRB3 $^{-1-}$ mice compared to $r d 16$ mice $(n=4-5)$. This preservation was in accordance with the improvement of a- and b-scotopic ERG amplitudes registered at P18 ( $n=3-7)$. C Images of the scotopic ERG traces. D ERG analysis demonstrated a statistically significant increase in the $\mathrm{a}$ - and $\mathrm{b}_{\text {-wave }}$ amplitudes in $\mathrm{rd}^{16} \mathrm{TRB}^{-1-}$ retinas. Data are shown as mean \pm SEM; ${ }^{* * * *} p<0.0001$. OS outer segments of photoreceptors, IS inner segments of photoreceptors, ONL outer nuclear layer, OPL outer plexiform layer, INL inner nuclear layer, IPL inner plexiform layer, RGC layer retinal ganglion cell layer.

learn about how mTOR activity is boosted in $r d 16 \mathrm{TRB}^{-/-}$retinas, we initiated a comparative study to evaluate autophagyassociated protein expression. We found that levels of Beclin-1, a protein involved in the initial step of phagophore formation, increased in $r d 16$ retinas compared to $\mathrm{C} 57 \mathrm{BL} / 6$ and $r d 16 \mathrm{TRB}^{-1-}$ mice (Fig. 4A, B, $p<0.05$ ). The same pattern was observed for ATG5 protein in $r d 16$ retinas when compared to $\mathrm{C} 57 \mathrm{BL} / 6$ retinas $(p<0.05)$. Because of the increase in ATG5, a protein influencing the growing phagophore and LC3 integration, we next determined the LC3 processing and measured non-lipidated and lipidated forms. We found that the LC3-II/I ratio was significantly increased in $r d 16$ mice compared to $\mathrm{C} 57 \mathrm{BL} / 6$ and $r d 16 \mathrm{TRB}^{-/-}$ retinas $(p<0.05)$, suggesting that LC3 II degradation by autophagosome may be impaired. Furthermore, the level of SQSTM1/p62, a selective autophagy receptor and a link between LC3 and ubiquitinated substrates, was also increased in $r d 16$ retinas $(p<$ 0.05). Together with LC3 II accumulation, the increase in p62 level indicates that autophagy flux may be impaired and should be carefully examined in degenerating retinas. To investigate further, we assessed the level of LAMP2, a lysosome-associated membrane protein-2 and an important regulator of autophagy. We found that rd16 retinas manifested a reduced level of LAMP2 compared to 


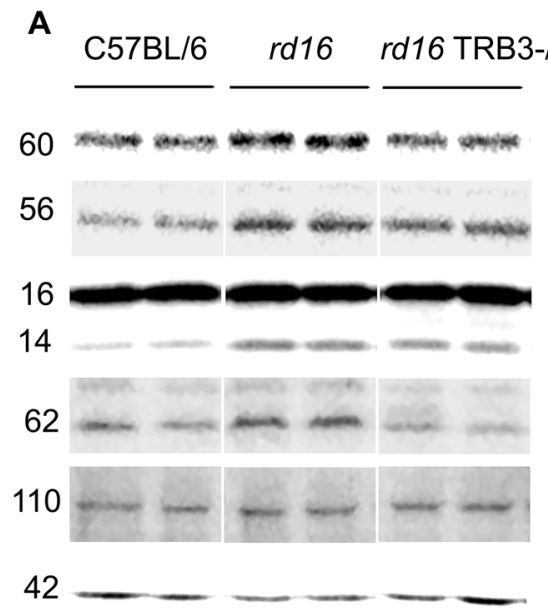

C

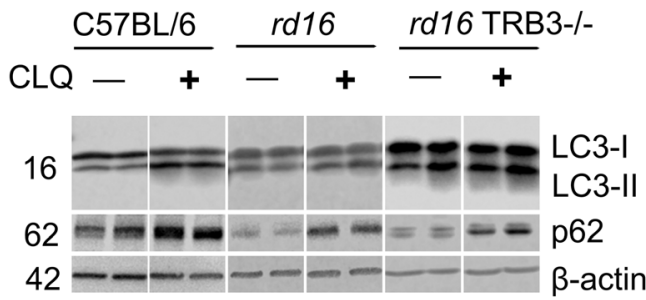

E

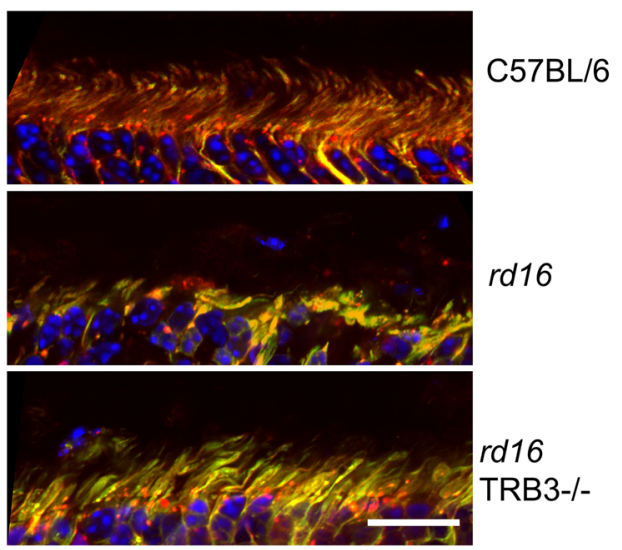
Beclin-1
ATG5
LC3-I
LC3-II
p62
LAMP2

B-actin
B
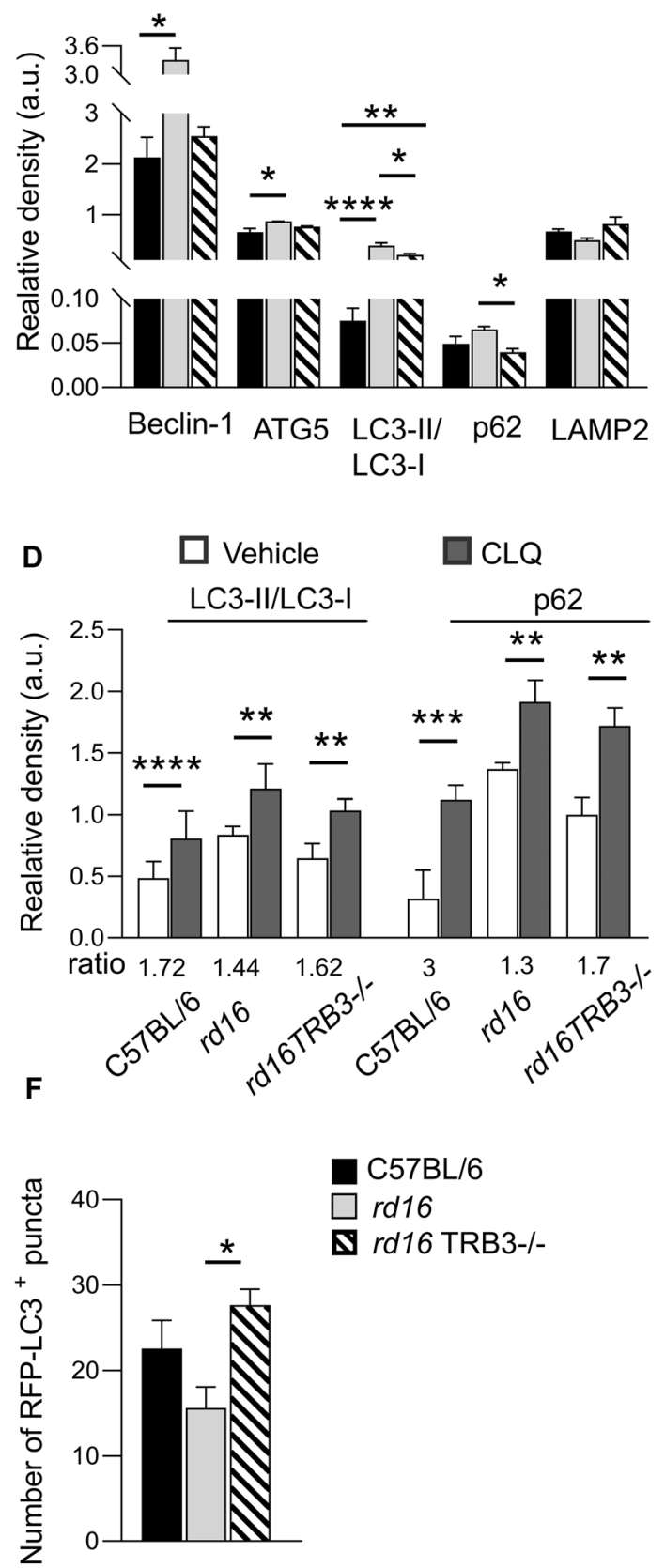

Fig. 4 TRB3 ablation in P15 rd16 retinas resulted in restoration of autophagy-associated protein levels and flux. A Images of western blot membranes probed with anti-Beclin-1, ATG5, LC3, p62, and LAMP2 antibodies. B Quantitation of Beclin-1, ATG5, LC3 II/I, p62, and LAMP2 in protein retinal extracts from three groups of mice $(n=4-5)$. C, D Treatment of retinal explants with chloroquine (CLQ), a classic inhibitor of autophagy, was used to determine the capacity of degenerating retinas to promote autophagosome fusion with lysosomes. C Images of western blot analysis demonstrated LC3 conversion and p62 accumulation. D Quantitation of LC3 conversion in CLQ-treated retinas $(n=6)$. The LC3 II/I ratios of the treated retinas were normalized to those of untreated retinas. An increase in induced LC3 conversion was detected in the treated $\mathrm{C} 57 \mathrm{BL} / 6$ and $r d 16 \mathrm{TRB}^{-1-}$ retinas. We also observed an increase in p62 level in treated and normalized rd16 retinas as compared to $\mathrm{C} 57 \mathrm{BL} / 6$ and $r d 16 \mathrm{TRB}^{-1-}(n=4-5)$. E, F The RFP-GFP-LC3 transgene expression in mouse retinas demonstrated the generation of red puncta in the inner segments of photoreceptors. E Images of the retinal sections of $\mathrm{C} 56 \mathrm{BL} / 6, r d 16$, and $r d 16 \mathrm{TRB}^{-/-}$mice taken with a confocal microscope. F Quantitation of the red puncta cells in the retinal sections of the three groups of mice $(n=5-7)$. Data are shown as mean \pm SEM; ${ }^{*} p<0.05,{ }^{* *} p<0.01,{ }^{* * *} p<0.001$, and ${ }^{* * * *} p<0.0001$.

C57BL/6 mice detected by western blot analysis, but this reduction was not statistically different.

Given that LC3-II/I conversion is increased in rd16 retinas, we next treated degenerating retinas with chloroquine (CLQ), a classic inhibitor of autophagy, to determine the capacity of autophagosomes in degenerating retinas to bind and deliver LC3 II to lysosomes (Fig. 4C, D). We observed that the LC3 conversion from I to II was limited in the CLQ-treated $r d 16$ mice, while the treated 
rd16 $\mathrm{TRB}^{-/-}$retinas-although to the degree observed in the $\mathrm{C} 57 \mathrm{BL} / 6$ retinas-demonstrated an increase in the conversion step compared to the untreated retinas. Thus, the LC3-II/I ratio in the treated mice compared with the ratio in the untreated C57BL/ 6 and $r d 16 \mathrm{TRB}^{-1-}$ retinas was 1.73 and 1.62 , respectively, compared to $r d 16$ retinas with a conversion index of only 1.44.

We next identified the p62 accumulation in treated compared with untreated retinas and found that the rd16 retinas demonstrated an impaired capacity to accumulate p62 under the CLQ treatment (Fig. 4D, E). The ratio of p62 in treated over untreated rd16 retinas was 1.3 , while for $\mathrm{C} 57 \mathrm{BL} / 6$ and $r d 16 \mathrm{TRB}^{-1-}$ retinas, the ratios were 3.0 and 1.7 , respectively.

Different transgenic mouse models may be leveraged to monitor various stages of autophagosome performance. One of these is the GFP-LC3 transgenic mouse, which has been widely used in the research field of retinal degeneration. In our study, we utilized mice expressing the RFP-GFP-LC3 reporter and conducted fluorescent microscopy at P15 to monitor positive RFP puncta in $\mathrm{C} 57 \mathrm{BL} / 6, r d 16$, and $r d 16 \mathrm{TRB}^{-1-}$ retinas expressing transgene (Fig. $4 \mathrm{E}, \mathrm{F})$. The results of the study indicated that the RFP signal, serving as a marker of autolysosomes, was generated in the ISs of photoreceptors. Counting the RFP-positive puncta in the ISs revealed that $r d 16$ mice had less formed autophagosolysosome fusion compared to rd16 $\mathrm{TRB}^{-1-}$ photoreceptors (Fig. 4E, F). Altogether, these data indicated an increase in the autophagyassociated protein levels and impaired autophagolysosome function in $r d 16$ retinas, while an improvement of autophagy flux in rd16 retinas was observed with TRB3 ablation.

\section{rd16 $\mathrm{TRB}^{-1-}$ retinas demonstrated an increase in the level of total protein ubiquitination and E3 ligases}

Recently, it has been highlighted that many neurodegenerative disorders share a common pathological feature, that is, accumulation of intracellular ubiquitin-positive inclusions formed by aggregate-prone neurotoxic proteins [33]. A study conducted in Arshavsky's laboratory demonstrated that the increase in ubiquitin proteasomal system (UPS) activity supports photoreceptor survival in IRD [34]. Because mTOR inhibition activates overall protein degradation by the UPS system as well as by autophagy [35], we next decided to analyze the level of total ubiquitinated (Ub) proteins. Surprisingly, western blot analysis demonstrated that the level of Ub proteins was reduced in the $r d 16$ retinas, while in the rd16 TRB3 $^{-1-}$ retinas, it was restored to the level observed in the C57BL/6 retinas (Fig. 5A, B). These results are in accord with the fact that the autophagy flux detected in earlier experiments was impaired in rd16 retinas; both the selective autophagy and the UPS utilize tagged proteins for further degradation in cells. Additionally, these data made us wonder how a major photoreceptor-specific rhodopsin protein is ubiquitinated and degraded in $r d 16$ retinas.

A properly folded rhodopsin is known to traffic to the outer segments (OS) of photoreceptors and be degraded by phagocytosis in the RPE cells. This process consists of multiple steps, including recognition by the RPE cells, binding, ingestion, phagosome maturation, and degradation of phagocytosed material [36]. Moreover, the interruption of any of these steps, such as the defect in OS development observed in rd16 retinas, could lead to the accumulation of unprocessed material inside the retina and eventually to retinal degeneration. As evidence, we observed the accumulation of rhodopsin in the ONL of $r d 16$ retinas at P20 (Fig. 1F). At P15, we subjected $r d 16$ retinal extracts to IP with 1D4 antibody and conducted western blot analysis; we found that the Ub-rhodopsin level was significantly increased in rd16 retinas compared to C57BL/6 and $r d 16 \mathrm{TRB}^{-1-}$ retinas (Fig. $5 C, D)$. Of note is that, in earlier experiments, the same $r d 16$ retinas (Fig. 1E, G) demonstrated the lowest level of total rhodopsin among the three groups, suggesting the majority of synthetized rhodopsin is ubiquitinated in degenerating retinas. Overall, these findings suggest that mTOR inhibition observed in $r d 16$ retinas correlates with the increase in the Ub-rhodopsin level. Keeping in mind these facts, we next asked how rhodopsin degrades in rd16 retinas. We tested the hypothesis that Ub-rhodopsin could be targeted by selective autophagy degradation, and rhodopsin is accumulated in the retina in response to treatment with CQL. We found no difference in the rhodopsin level in retinas treated with autophagy inhibitor (data not shown), noting that that in $r d 16$ retinas, Ub-rhodopsin degrades through UPS.

Because of the enhanced rhodopsin ubiquitination, we next wondered whether E3 ligases are altered in degenerating retinas. Among all the known ligases present in the retina, we chose to test UBR1, which has been reported to be responsible for rhodopsin degradation [37]. We observed no difference in UBR1 expression in both retinal degenerative groups, suggesting that at P15, mice manifesting ciliopathy, although they have different levels of Ub-rhodopsin, have similar UBR1 levels (data not shown).

Unlike the in vivo results, TRB3 controlled UBR1 expression in the human MIO-M1 cells (Fig. 5G, H). The sequential transfection of cells with plasmid expressing TRB3 and ASO-TRB3 demonstrated that, compared to cells transfected with ASO-control, there was a significant restoration of UBR1. Therefore, the in vitro study indicated a potential TRB3-mediated control of E3 UBR1 ligase expression.

We next analyzed the level of E3 Parkin 1 ligase, which has been reported to be a downstream TRB3 target, the expression of which is inhibited by TRB3 [38] (Fig. 5E, F). Indeed, a dramatic increase in Parkin 1 level was detected in rd16 deficient in TRB3, while rd16 retinas maintained the basal level of E3 ligase. Overall, these data indicate that the UPS may operate more efficiently in $r d 16 \mathrm{TRB}^{-1-}$ retinas compared to rd16 retinas, and this activation occurs concomitantly with improvement in autophagy flux and an increase in protein synthesis in these mice at P15.

\section{DISCUSSION}

ISR is involved in retinal pathogenesis in various retinal degenerative diseases. In this study, we presented evidence that IRS-induced TRB3 overexpression in rd16 retinas is responsible for the progression of retinal dystrophy. First, we found that TRB3 regulates $A K T / m T O R$ phosphorylation in the retina. Then, we learned that TRB3 ablation is responsible for the restoration of AKT/mTOR activity in these mice. The restoration of $p$-mTOR results in photoreceptor cell survival assessed by functional and morphological analyses and serves as a homeostatic rheostat of major cellular pathways in the retina.

The pseudokinase TRB3 regulates AKT phosphorylation. Cell culture experiments that employed ASOs targeting TRB3 have demonstrated direct TRB3-mediated control of AKT activity in Müller cells. Both the upregulation of TRB3 and the decline in pAKT and p-mTOR accompanied by a diminished translational rate occurred in rd16 mice at P15 [19]. Moreover, the level of deactivated (phosphorylated) translational repressor 4E-BP1 controlled by mTOR was lower in $r d 16$ retinas compared to C57BL/6 mice. Previously, we found that the regulation of the activity of the translational repressor 4E-BP1 plays an essential role in the 5'-cap translational mechanism in degenerating retinas, and the longlasting translational attenuation during the course of retinal degeneration results in adverse effects on the photoreceptors [27]. In the current study, we observed that the functional and morphological rescue of $r d 16 \mathrm{TRB}^{-1-}$ photoreceptors associated with AKT/mTOR restoration is accompanied by the repair of the translational rate and an increase in rhodopsin protein.

Numerous studies conducted in various in vitro and in vivo systems have demonstrated that mTOR plays a crucial role in regulating autophagy. For example, mTOR is known to inhibit the VPS34 complex, which includes Beclin as a partner [32]. Therefore, it is no surprise that in our study, rd16 retinas with diminished 


\section{A}

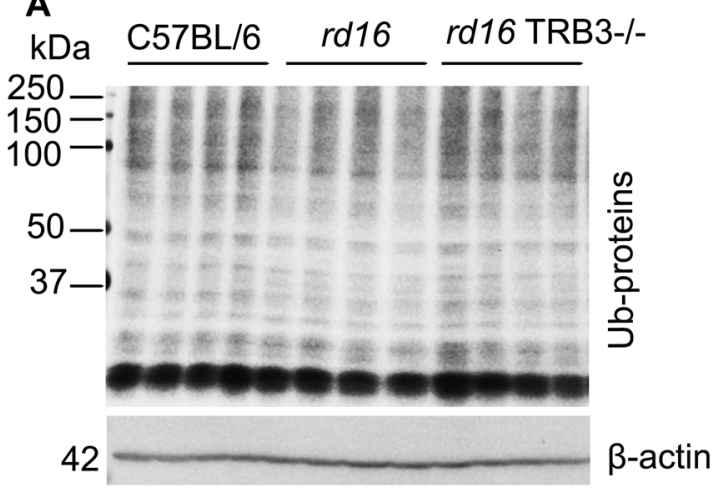

C

kDa C57BL/6 rd16 rd16 TRB3-/-

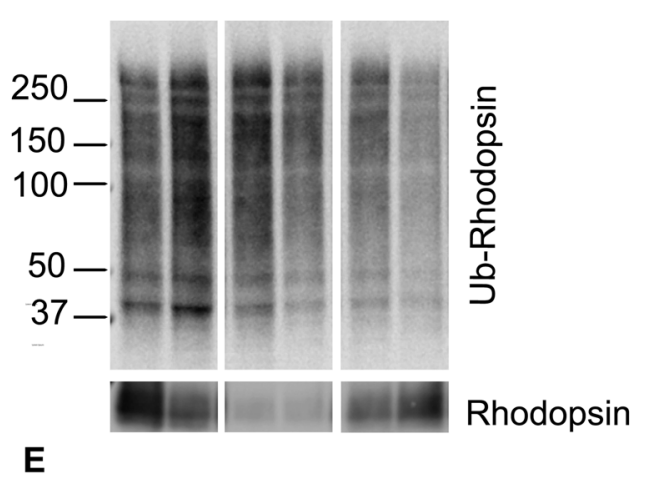

kDa C57BL/6 rd16 rd16 TRB3-/-

50

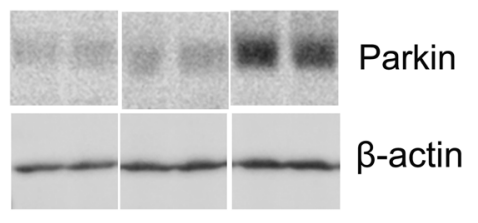

G

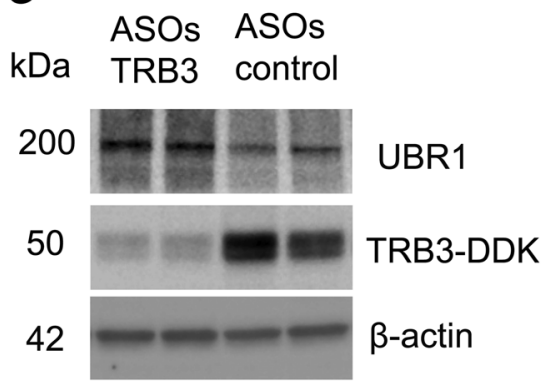

B

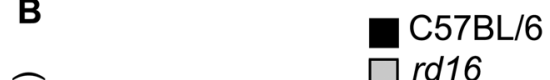

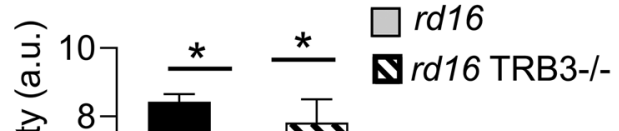

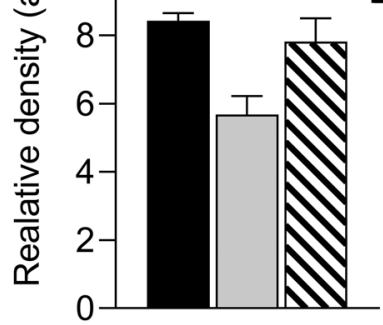

Ub-proteins

D

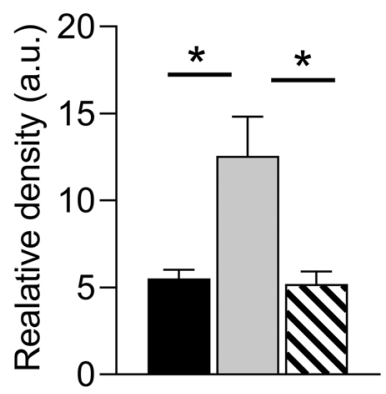

Ub-Rhodopsin

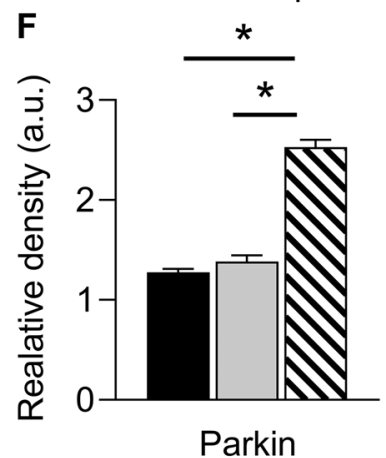

H

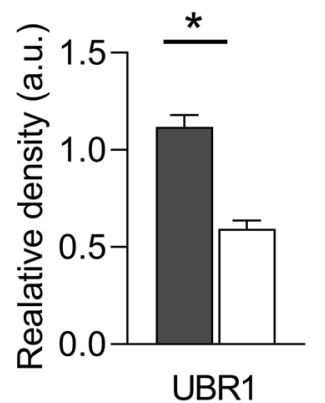

ASOs TRB3

ASOs control

Fig. 5 TRB3 ablation in rd16 retinas led to the restoration of the ubiquitinated protein level and an increase in E3 ligase expression. A Images of western blot membrane probed with anti-Ub antibody to detect level of total protein ubiquitination. B Calculation of the band density measured for western blot (A). Significant reduction in the total Ub-protein levels was detected in $r d 16$ retinas $(n=8)$. C, D The Ubrhodopsin level was detected by performing the IP reaction using protein extracts from $\mathrm{C} 56 \mathrm{BL} / 6, r d 16$, and $r d 16 \mathrm{TRB}{ }^{-1-}$ mice. C Images of western blot probed with anti-Ub and Rhodopsin antibodies. D Levels of Ub-rhodopsin from IP reactions were normalized through total rhodopsin protein. Measurement indicated that rhodopsin protein was highly ubiquitinated in the $r d 16$ retinas. E, $\mathbf{F}$ Parkin 1 ligase expression in the retinas of C56BL/6, $r d 16$, and $r d 16 \mathrm{TRB}^{-1-}$ mice and MIO-M1 Müller cells $(n=4)$. E Images of western blot obtained with retinal protein extracts from P15 C56BL/6, rd16, and $r d 16 \mathrm{TRB}^{-/-}$mice probed with anti-Ubr1, Parkin 1, and $\beta$-actin antibodies. F TRB3 ablation in $r d 16$ resulted in increase in Parkin 1 protein compared to both the C57BL/6 and $r d 16$ mice. G, H Sequential transfection of Müller cells with plasmid expressing TRB3 CDNA and ASO-TRB3 $(n=4)$. G Images of western blots running with protein extracts probed with anti-UBR1, TRB3, and $\beta$-actin antibodies. H Quantitation of UBR1 expression in cells co-transfected with ASO-control and ASO-TRB3. A significant decrease in UBR1 level was observed in cells transfected with ASO-TRB3. Data are shown as mean \pm SEM; ${ }^{*} p<0.05,{ }^{* *} p<0.01,{ }^{* *} p<0.001$, and ${ }^{* * *} p<0.0001$. 
mTOR showed upregulation of Beclin-1 and ATG5 proteins and, contrarily, rd16 $\mathrm{TRB}^{-1-}$ retinas with a restored AKT/mTOR axis manifest autophagy protein levels comparable with C57BL/6 retinas. However, it should also be mentioned that $r d 16$ retinas manifested impaired autophagy flux; the LC3 II and p62 accumulations in $r d 16$ retinas provided evidence of autophagic dysfunction. The treatment of retinal explants with CQL shows that $r d 16$ retinas have less "room" for conversion of LC3 I to II, indicating the original problems with LC3 lipidation in forming LC3 II occurring in these mice.

Not only is autophagosome formation impaired in $r d 16$ retinas but their fusion with lysosomes is affected, as well. We found that the number of RFP-LC3 in photoreceptors' autolysosomes declined in $r d 16$ mice and increased in $r d 16 \mathrm{TRB}^{-1-}$ retinas, indicating the TRB3 mediated control of autophagolysosome fusion. Overall, the application of the RFP-GFP-LC3 mice allowed us to count the autophagolysosome fusion in photoreceptors. However, it is worth mentioning that we need to be cautious about leveraging RFP-GFP-LC3 mice expressing mutant photoreceptor-specific genes that lead to the rapid onset of retinal degeneration. P15 rd16 mice manifest altered photoreceptor cell morphology with shortening of ISs. This fact may introduce an error in the counting of red puncta cells, which suggests that fluorescence microscopy should be conducted when the ISs are well-preserved in the degenerating retina. However, these time points may not correlate with the rate of autophagy flux dysfunction.

The total Ub-protein level is diminished in $r d 16$ retinas, whereas it is restored in $r d 16 \mathrm{TRB}^{-1-}$ mice. Since both autophagy and the UPS utilize Ub-labeled proteins, and a compensatory balance between their activities exists in mammalian cells to maintain cellular homeostasis [39], rd16 retinas may clear Ub-protein less efficiently due to diminished autophagosomes and preferential degradation of Ub-protein by UPS. For example, we found that at P15, when rd16 retinas manifest a low level of rhodopsin, the majority of rhodopsin is ubiquitinated. Given our results evidencing no rhodopsin degradable by autophagy, it seems that UPS activity is sufficient to support photoreceptors at this point, and no rhodopsin accumulation was observed in the retina at P15 [27]. However, at later time points (Fig. 1F), we observed rhodopsin staining in the ONL in $r d 16 \mathrm{TRB}^{-/-}$retinas, which could be due to a few reasons: (1) an increase in rhodopsin synthesis, (2) mistrafficking of rhodopsin due to the lack of OS of photoreceptors, and (3) accumulation of rhodopsin due to impaired degradation.

TRB3 is known to regulate autophagy and degradation overall. TRB3 interacts with p62 and impedes p62 autophagic/proteasomal degradation [40]; disrupting the TRB3-p62 interaction is known to restore autophagy [41]. Therefore, TRB3-mediated p62 accumulation could be a reason for the impaired autophagolysosome formation in $r d 16$ retinas. This also explains why the $r d 16$ $\mathrm{TRB}^{-1-}$ retinas demonstrated a decrease in p62 accumulation. Overall, these data support earlier proposals that TRB3 overexpression constrains autophagy and that TRB3 knockdown induces autophagy flux [42].

The TRB3 molecule has been reported to regulate degradation through association with E3 COP1 [43], Mindbomb [44], and Parkin 1 ligases [45]. In addition, this protein can activate deubiquitinase USP9x [44] expression in the retina [46] and, therefore, reduce the accumulation of Ub-protein in $r d 16$ retinas. In our study, we found that TRB3 ablation resulted in a dramatic upregulation of E3 ligase Parkin 1 in $r d 16$ retinas. In MIO-M1 Müller cells, we also found that UBR1 ligase was controlled by TRB3 expression. On the other hand, the inhibition of mTOR in rd16 retinas could activate the degradation of proteins independent of TRB3 overexpression. Zhao et al. [35] reported that a rapid increase in UPS-mediated proteolysis resulting primarily from $\mathrm{mTORC} 1$ inhibition could occur without the requirement of new protein synthesis or key
mTOR targets such as $\mathrm{S} 6 \mathrm{Ks}$, 4E-BPs, or Ulks. These data suggest that TRB3-mediated signaling during the course of IRD directly or indirectly inhibits a clearance of Ub-proteins.

In conclusion, we validated TRB3 as a new therapeutic target to delay photoreceptor cell degradation and functional loss. Collectively, these data demonstrate that TRB3 regulates the AKT/mTOR axis in the retina and impacts AKT/mTOR downstream signaling, including 5'cap-dependent translation, autophagy, and UPS-dependent degradation. Therefore, approaches based on the application of ASOs or interference RNAs as well as on cell penetrating peptides to reduce either the expression or activity of TRB3 should be taken into consideration in the treatment of IRD.

\section{REFERENCES}

1. Gorbatyuk MS, Starr CR, Gorbatyuk OS. Endoplasmic reticulum stress: new insights into the pathogenesis and treatment of retinal degenerative diseases. Prog Retin Eye Res. 2020;79:100860.

2. Wubben TJ, Pawar M, Smith A, Toolan K, Hager H, Besirli CG. Photoreceptor metabolic reprogramming provides survival advantage in acute stress while causing chronic degeneration. Sci Rep. 2017;7:17863.

3. Maiese K. Molecules to medicine with mTOR: translating critical pathways into novel therapeutic strategies. Amsterdam: Elsevier/Academic Press; 2016.

4. Santini E, Heiman M, Greengard P, Valjent E, Fisone G. Inhibition of mTOR signaling in Parkinson's disease prevents L-DOPA-induced dyskinesia. Sci Signal. 2009;2:ra36.

5. Decressac M, Bjorklund A. mTOR inhibition alleviates L-DOPA-induced dyskinesia in parkinsonian rats. J Parkinsons Dis. 2013;3:13-7.

6. Ravikumar B, Vacher C, Berger Z, Davies JE, Luo S, Oroz LG, et al. Inhibition of mTOR induces autophagy and reduces toxicity of polyglutamine expansions in fly and mouse models of Huntington disease. Nat Genet. 2004;36:585-95.

7. Lee JH, Tecedor L, Chen YH, Monteys AM, Sowada MJ, Thompson LM. et al. Reinstating aberrant mTORC1 activity in Huntington's disease mice improves disease phenotypes. Neuron. 2015;85:303-15.

8. Park HL, Kim JH, Park CK. Different contributions of autophagy to retinal ganglion cell death in the diabetic and glaucomatous retinas. Sci Rep. 2018;8:13321.

9. Zhao C, Vollrath D. mTOR pathway activation in age-related retinal disease. Aging (Albany NY). 2011;3:346-7.

10. Chen Y, Wang J, Cai J, Sternberg P. Altered mTOR signaling in senescent retinal pigment epithelium. Invest Ophthalmol Vis Sci. 2010;51:5314-9.

11. Huang Y, Chen Y, Shaw AM, Goldfine H, Tian J, Cai J. Enhancing TFEB-mediated cellular degradation pathways by the mTORC1 inhibitor Quercetin. Oxid Med Cell Longev. 2018;2018:5073420.

12. Yagasaki R, Nakahara T, Mori A, Sakamoto K, Ishii K. Effects of mTOR inhibition on normal retinal vascular development in the mouse. Exp Eye Res. 2014;129:127-34.

13. Rajala A, Wang Y, Rajala RVS. Constitutive activation mutant mTOR promote cone survival in retinitis pigmentosa mice. Adv Exp Med Biol. 2018;1074:491-7.

14. Punzo $C$, Cepko $C$. Cellular responses to photoreceptor death in the rd1 mouse model of retinal degeneration. Invest Ophthalmol Vis Sci. 2007;48:849-57.

15. Zieger M, Punzo C. Improved cell metabolism prolongs photoreceptor survival upon retinal-pigmented epithelium loss in the sodium iodate induced model of geographic atrophy. Oncotarget. 2016;7:9620-33.

16. Petit L, Punzo C. mTORC1 sustains vision in retinitis pigmentosa. Oncotarget. 2015;6:16786-7.

17. Venkatesh A, Ma S, Le YZ, Hall MN, Ruegg MA, Punzo C. Activated mTORC1 promotes long-term cone survival in retinitis pigmentosa mice. J Clin Investig. 2015;125:1446-58.

18. Zhang L, Justus S, Xu Y, Pluchenik T, Hsu CW, Yang J, et al. Reprogramming towards anabolism impedes degeneration in a preclinical model of retinitis pigmentosa. Hum Mol Genet. 2016;25:4244-55.

19. Starr CR, Pitale PM, Gorbatyuk M. Translational attenuation and retinal degeneration in mice with an active integrated stress response. Cell Death Dis. 2018;9:484.

20. Du K, Herzig S, Kulkarni RN, Montminy M. TRB3: a tribbles homolog that inhibits Akt/PKB activation by insulin in liver. Science. 2003;300:1574-7.

21. Borsting E, Patel SV, Declèves AE, Lee SJ, Rahman QM, Akira $S$, et al. Tribbles homolog 3 attenuates mammalian target of rapamycin complex-2 signaling and inflammation in the diabetic kidney. J Am Soc Nephrol. 2014;25:2067-78.

22. Ohoka N, Yoshii S, Hattori T, Onozaki K, Hayashi H. TRB3, a novel ER stressinducible gene, is induced via ATF4-CHOP pathway and is involved in cell death. EMBO J. 2005;24:1243-55. 
23. Ord T, Ord T. Mammalian pseudokinase TRIB3 in normal physiology and disease: charting the progress in old and new avenues. Curr Protein Pept Sci. 2017;18:819-42.

24. Xing Y, Luo P, Hu R, Wang D, Zhou G, Jiang J. TRIB3 promotes lung adenocarcinoma progression via an enhanced Warburg effect. Cancer Manag Res. 2020;12:13195-206.

25. Satoh T, Kidoya H, Naito H, Yamamoto M, Takemura N, Nakagawa K, et al. Critical role of Trib1 in differentiation of tissue-resident M2-like macrophages. Nature. 2013;495:524-8.

26. Yoshii SR, Mizushima N. Monitoring and measuring autophagy. Int J Mol Sci. 2017;18:1865. https://doi.org/10.3390/ijms18091865.

27. Starr CR, Nyankerh CNA, Qi X, Hu Y, Gorbatyuk OS, Sonenberg N, et al. Role of translational attenuation in inherited retinal degeneration. Invest Ophthalmol Vis Sci. 2019;60:4849-57.

28. Limb GA, Salt TE, Munro PM, Moss SE, Khaw PT. In vitro characterization of a spontaneously immortalized human Muller cell line (MIO-M1). Invest Ophthalmol Vis Sci. 2002;43:864-9.

29. Weismann D, Erion DM, Ignatova-Todorava I, Nagai Y, Stark R, Hsiao JJ, et al. Knockdown of the gene encoding Drosophila tribbles homologue 3 (Trib3) improves insulin sensitivity through peroxisome proliferator-activated receptorgamma (PPAR-gamma) activation in a rat model of insulin resistance. Diabetologia. 2011;54:935-44

30. Hao F, Itoh T, Morita E, Shirahama-Noda K, Yoshimori T, Noda T. The Ptdlns3phosphatase MTMR3 interacts with mTORC1 and suppresses its activity. FEBS Lett. 2016;590:161-73.

31. Shinde V, Kotla P, Strang C, Gorbatyuk M. Unfolded protein response-induced dysregulation of calcium homeostasis promotes retinal degeneration in rat models of autosomal dominant retinitis pigmentosa. Cell Death Dis. 2016;7: e2085.

32. Kim YC, Guan KL. mTOR: a pharmacologic target for autophagy regulation. J Clin Invest. 2015;125:25-32.

33. Zheng $Q$, Huang $T$, Zhang $L$, Zhou $Y$, Luo $H, X u H$, et al. Dysregulation of ubiquitin-proteasome system in neurodegenerative diseases. Front Aging Neurosci. 2016;8:303.

34. Lobanova ES, Finkelstein S, Li J, Travis AM, Hao Y, Klingeborn M, et al. Increased proteasomal activity supports photoreceptor survival in inherited retinal degeneration. Nat Commun. 2018;9:1738.

35. Zhao J, Zhai B, Gygi SP, Goldberg AL. mTOR inhibition activates overall protein degradation by the ubiquitin proteasome system as well as by autophagy. Proc Natl Acad Sci USA. 2015:112:15790-7.

36. Gordiyenko NV, Fariss RN, Zhi C, MacDonald IM. Silencing of the CHM gene alters phagocytic and secretory pathways in the retinal pigment epithelium. Invest Ophthalmol Vis. Sci. 2010;51:1143-50.

37. Ozawa Y, Nakao K, Kurihara T, Shimazaki T, Shimmura S, Ishida S, et al. Roles of STAT3/SOCS3 pathway in regulating the visual function and ubiquitinproteasome-dependent degradation of rhodopsin during retinal inflammation. J Biol Chem. 2008;283:24561-70.

38. Aimé $P$, Sun X, Zareen N, Rao A, Berman Z, Volpicelli-Daley L, et al. Trib3 is elevated in Parkinson's disease and mediates death in Parkinson's disease models. J Neurosci. 2015;35:10731-49.

39. Kocaturk NM, Gozuacik D. Crosstalk between mammalian autophagy and the ubiquitin-proteasome system. Front Cell Dev Biol. 2018;6:128.

40. Hua F, Li K, Yu JJ, Lv XX, Yan J, Zhang XW, et al. TRB3 links insulin/IGF to tumour promotion by interacting with $\mathrm{p} 62$ and impeding autophagic/proteasomal degradations. Nat Commun. 2015;6:7951.

41. Zhang XW, Zhou JC, Peng D, Hua F, Li K, Yu JJ, et al. Disrupting the TRIB3-SQSTM1 interaction reduces liver fibrosis by restoring autophagy and suppressing exosome-mediated HSC activation. Autophagy. 2020;16:782-96.

42. Tang Z, Chen H, Zhong D, Wei W, Liu L, Duan Q, et al. TRIB3 facilitates glioblastoma progression via restraining autophagy. Aging (Albany NY). 2020;12:25020-34. Epub 2020 Nov 16 https://doi.org/10.18632/aging.103969.

43. Qi L, Heredia JE, Altarejos JY, Screaton R, Goebel N, Niessen S, et al. TRB3 links the E3 ubiquitin ligase COP1 to lipid metabolism. Science. 2006;312:1763-6.
44. Izrailit J, Jaiswal A, Zheng W, Moran MF, Reedijk M. Cellular stress induces TRB3/ USP9x-dependent Notch activation in cancer. Oncogene. 2017;36:1048-57.

45. Yang Y, Nishimura I, Imai Y, Takahashi R, Lu B. Parkin suppresses dopaminergic neuron-selective neurotoxicity induced by Pael-R in Drosophila. Neuron 2003;37:911-24.

46. Esquerdo-Barragan M, Brooks MJ, Toulis V, Swaroop A, Marfany G. Expression of deubiquitinating enzyme genes in the developing mammal retina. Mol Vis. 2019;25:800-13.

\section{ACKNOWLEDGEMENTS}

We thank luliia Dorofeeva for technical assistance in the assay of this study.

\section{AUTHOR CONTRIBUTIONS}

IVS, AE, PMP, and OSG performed experiments and analyzed data. IVS, MA, and MSG designed experiments and interpreted data; IVS and MSG wrote the manuscript; and MSG finalized the manuscript.

\section{FUNDING STATEMENT}

This work was supported by the National Eye Institute, grants R01 EY027763 and R21YE031103.

\section{COMPETING INTERESTS}

The authors declare no competing interests.

\section{ETHICS}

Experiments performed with animals compiled with the guidelines of the Association of Research in Vision Science and Ophthalmology and were approved by the Institutional Animal Car and Use Committee (IACUC) of the University of Alabama at Birmingham.

\section{ADDITIONAL INFORMATION}

Correspondence and requests for materials should be addressed to M.S.G.

Reprints and permission information is available at http://www.nature.com/ reprints

Publisher's note Springer Nature remains neutral with regard to jurisdictional claims in published maps and institutional affiliations.

Open Access This article is licensed under a Creative Commons Attribution 4.0 International License, which permits use, sharing, adaptation, distribution and reproduction in any medium or format, as long as you give appropriate credit to the original author(s) and the source, provide a link to the Creative Commons license, and indicate if changes were made. The images or other third party material in this article are included in the article's Creative Commons license, unless indicated otherwise in a credit line to the material. If material is not included in the article's Creative Commons license and your intended use is not permitted by statutory regulation or exceeds the permitted use, you will need to obtain permission directly from the copyright holder. To view a copy of this license, visit http://creativecommons. org/licenses/by/4.0/.

(C) The Author(s) 2021 